\title{
Software Defined Networking (SDN): A Revolution in Computer Network
}

\author{
Md. HumayunKabir \\ Department of Computer Science \& Engineering, University of Rajshahi, Rajshahi, Bangladesh.
}

\begin{abstract}
SDN creates a dynamic and flexible network architecture that can change as the business requirements change. The growth of the SDN market and cloud computing are very much connected. As the applications change and the network is abstracted, virtualization become a necessary step and SDN serves as the fundamental building blocks for the network. Traditional networking devices are composed of an embedded control plane that manages switching, routing and traffic engineering activities while the data plane forwards packet/frames based on traffic. In SDN architecture, control plane functions are removed from individual networking devices and embedded in a centralizedserver. The SDN controller makes all traffic related decisions in the network without nodes active participation, as opposed to today's networks.
\end{abstract}

Keyword-API, cloud computing, IT, middleware, OpenFlow, SDN

\section{Introduction}

Everyday new technology, new devices are emerging, todays networking concept is also changing accordingly. The traditional network infrastructure is a single system composed of many physical elements, such as routers, switches, and firewalls on which the entire IT portfolio depends for communication and services. A change in any part of the network can cause a failure of the whole. Most people recognize that traditional networking paradigms are too static and require a lot of effort to physically change and laboriously configure and validate the network [1].

SDN has appeared as a promising new approach to better handle the exponential growth in data traffic, network virtualization, and mobility[2] [3]. SDN allows network administrators/operators to control their networks programmatically, helping them to add capabilities and scale without compromising performance, reliability, or user experience [4].

\section{Traditional Networkto SDN Network}

The growing importance of the network has been a central theme in the modern communication. Everything now runs over a network and the network serves as the delivery and access vehicle. A traditional network layout (Fig.1) as it compares to an SDN network layout (Fig.2)is illustrated in the following.

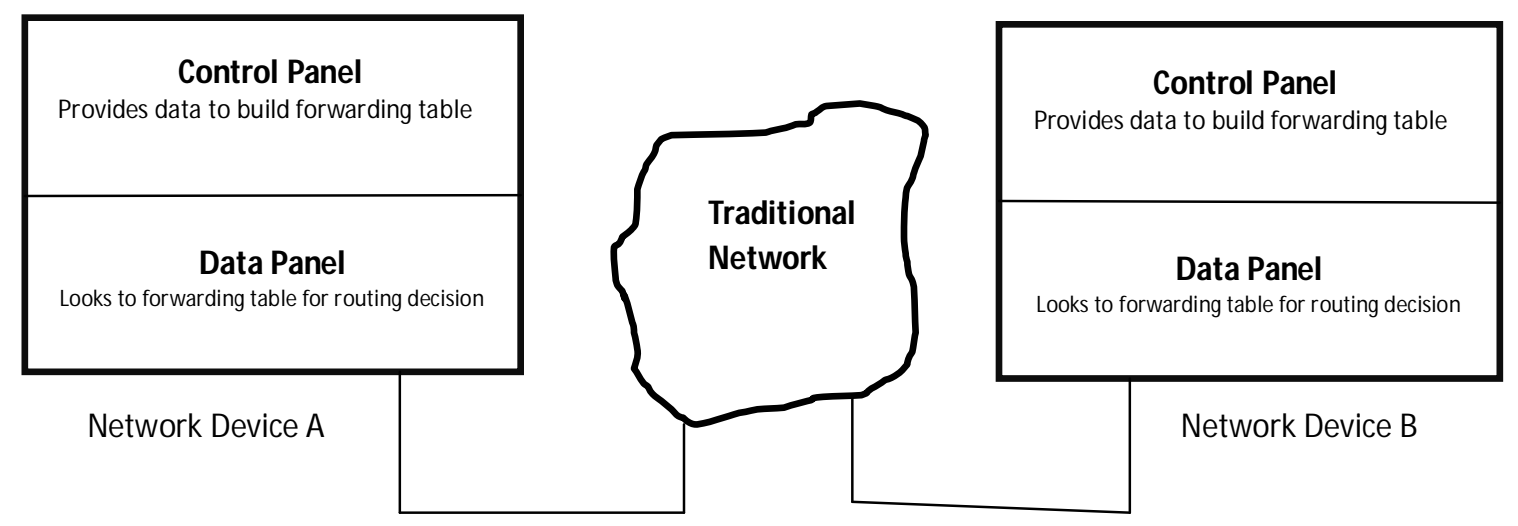

Figure 1: Traditional network layout

Traditional networking devicesare composed of an embedded control plane that manages switching, routing and traffic engineering activities while the data plane forwards packet/frames based on traffic [5]. Here control plane acts as the traffic manager and data plane as the traffic carrier. The control plane provides information used to build a forwarding table. The data plane consults the forwarding tableto make a decision on where to send frames or packets entering the device. Both of these planes are presented directly on the networking device [6]. 


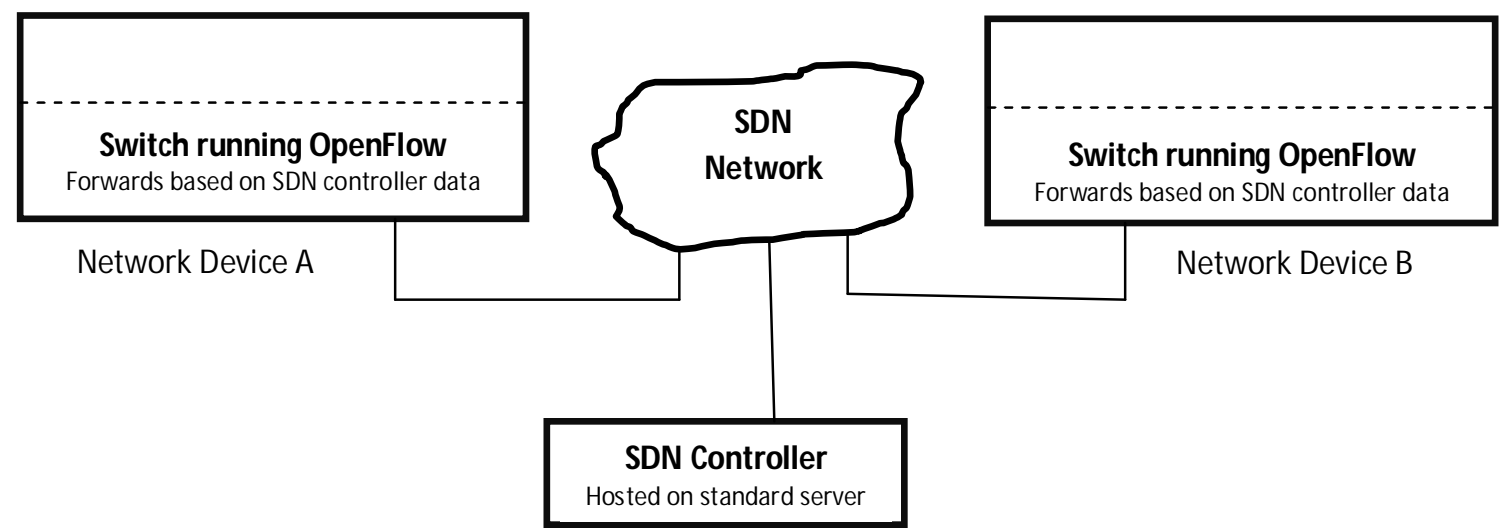

Figure 2:SDN network layout

In SDN architecture, control plane functions are removed from individual networking devices and hostedon a centralizedserver [7]. The SDN controller can be a server running SDN software. The controller communicates with a physical or virtual switch data plane through a protocol called OpenFlow [8]. OpenFlow carries the instructions to the data plane on how to forward data. The network devices must run the OpenFlow protocol for this to be possible.

\section{LimitationsofTraditionalNetworking}

Current network architectures partially meet only today's requirements of enterprises, carriers, and end users [9]. In today's networking, many discrete sets of protocols are used to connect hosts reliably over arbitrary distances, link speeds, and topologies. Protocols are defined in isolation, to solve a specific problem. There is almost no benefit of any fundamental abstractions. To add or move any device, IT must (re)configure multiple hardware/software entities using device-level management tools by considering topology, vendor switch model, software version, etc. Moreover, today's networks reconfigurations are performed relatively in static way to minimize the risk of service disruption[10]. The static nature of networks is not good for today's dynamic server environment, server virtualization and virtual machines (VMs) migration. Applications are distributed across multiple VMs, which exchange traffic flows with each other. To implement network-wide policy IT have to configure thousands of devices and mechanisms. The complexity of today's networks makes it very difficult to apply a consistent set of access, security, QoS, and other policies [11].

\section{ONFReferenceModelfor SDN}

SDN is a new architecture that has been designed to enable more agile and cost-effective networks. The Open Networking Foundation (ONF) is taking the lead in SDN standardization, and has defined an SDN architecture model[7] [12-14] as depicted in Fig.3.

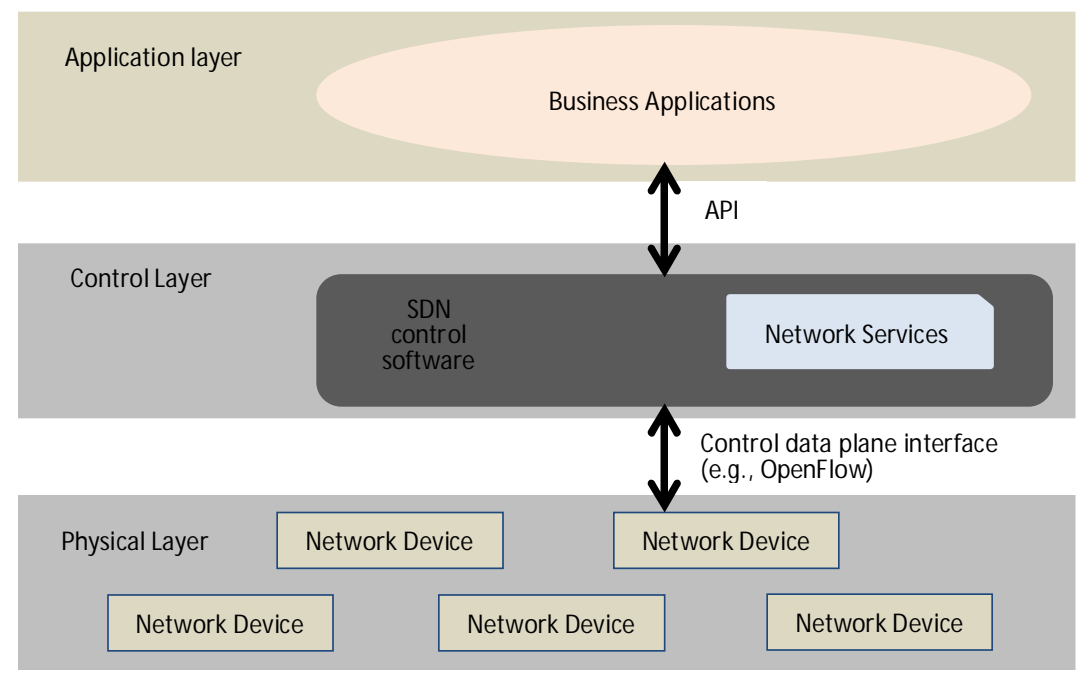

Figure 3: ONF SDN reference model 
The ONF/SDN architecture consists of three distinct layers that are accessible through open APIs:

- The application layer consists of the end-user business applications that provide different communications services. Communications between the application layer and the control layer ismanaged by the API.

- The control layer controlsand supervises the network forwarding functionality through an open interface.

- The physicallayer consists of the physical network devices (i.e. router, switch, etc.) that provide packet switching and forwarding.

According to this model, SDN architecture is characterized by three key attributes:

1. Logically centralized intelligence

An SDN provides global network orchestration from a single point of management using the standard interface OpenFlow. By centralizing network intelligence, decision-making is facilitated based on a global (or domain) view where nodes are unaware of the overall state of the network.

2. Programmability

An SDN offer programmatic interfaces that can automate and shape network fabric configuration. SDN networks can achieve revolution and differentiation from traditional network by providing open APIs for applications to communicate with the network.

3. Abstraction

In an SDN network, the business applications that consume SDN services are abstracted from the underlying network technologies. Network devices are also abstracted from the SDN control layer to ensure portability with any application from any vendor.

\section{Cloud Computingand SDN}

Cloud computing is all about "abstracting servers," turning actual hardware into virtual machines [15]. Probably SDN serve the same thing with networking. As cloud environments grow, the development and implementation of applications will change. To keep up with the speed of change on the cloud computing side, virtualization has become a necessary step for the network.

A cloud computing system is divided into two sections: the front end and the back end [16]. The front end includes the client's computer (or computer network) and the application required to access the cloud computing system. The back end of the system consists of the various computers, servers and data storage systems that create the "cloud" of computing services. A central server administers the system, monitoring traffic and client demands to ensure everything runs smoothly. It follows a set of rules called protocols and uses a special kind of software called middleware. Middleware allows networked computers to communicate with each other.

In SDN, the communications protocol, OpenFlow, perform the same things as middleware do in the cloud computing environment. By using an API, the controller can implement network commands to multiple devices without the need to learn the command line syntax of multiple vendor products. SDN is needed especially in the ability to program and automate in order to have the cloud scale. SDN is a play maker of the cloud scaling to thousands and tens of thousands of applications and/or servers per IT resource.

\section{SDNChallenges}

SDN promises to alleviate the limitations of traditional networks.

- Today's network is hardware dominated and different types of new devices are used to construct the network landscape that is being repositioned. Virtualized servers and storages are rapidly being introduced to the network, but traditional networks are unableto appreciate the full prospective of virtualization.

- SDN is emerged as an approach to make high-capacity networks cheaper to build and especially to reconfigure on the fly - as well as potentially faster and more efficient.

- SDN promises many capabilities and flexibility that are invisible in traditional networks; however, SDN is in infancy stage and its results are hardly tested.

\section{Conclusion}

SDN will centralize and simplify control of the network, make networks programmable and more agile, and create opportunities for policy-driven supervision and more automation. As more and more computing moves to the cloud, those network improvements will be critical to keeping everything affordable and available. It won't be just cloud environments that will get SDN benefits, also SDN could enable corporate networks to be reconfigured on-the-fly. In short, SDN will help networks keep up with the speed of change made possible by the virtualization of other data center resources and provide the perfect supplement to cloud computing.

\section{Future Scope}

SDN will be required in order to have the cloud scale, especially the ability to program and automate.As SDN promises to change network traffic into network flows, traditional networking will have to be 
changedbecause the technology behind them and their function in the network will change. To adopt and get the benefits of SDN controller peer-to-peer security mechanisms will have tobe established.

\section{Acknowledgements}

The author would like to thank his family membersfor their support.

\section{References}

[1] IBM Systems and Technology, Software Defined Networking - A new paradigm for virtual, dynamic, flexible networking, October 2012

[2] Kirk Bloede, Software Defined Networking - Moving Towards Mainstream, Electronics Banking Research, August 2012

[3] Brocade VCS Fabrics: The Foundation for Software-Defined Networks

[4] Network Transformation with Software-Defined Networking and Ethernet Fabrics, Brocade Communications Systems, Inc., 2012

[5] Software Defined Networking: What Is It and Why Do You Need It?enterasys secure network

[6] http://globalconfig.net/software-defined-networking-vs-traditional/

[7] Open Networking Foundation, Software-Defined Networking: The New Norm for Networks

[8] http://www.brocade.com/solutions-technology/technology/software-defined-networking/openflow.page

[9] EugenBorcoci, Software Defined Networking and Architectures, Netware 2013 Conference, August 25, 2013 Barcelona

[10] Juniper Networks, Cloud Ready data center network design guide

[11] Cisco Unified Access Technology Overview: Converged Access

[12] Open Networking Foundation,OpenFlow ${ }^{\mathrm{TM}}$-Enabled Mobile and Wireless Networks, ONF Solution Brief, September 30, 2013

[13] Open Networking Foundation, SDN in the Campus Environment, ONF Solution Brief September 30, 2013

[14] Open Networking Foundation, SDN Security Considerations in the Data Center, ONF Solution Brief October 8, 2013

[15] http://readwrite.com/2013/04/23/software-defined-networking-sdn\#awesm= opb9QdgnFbp9T6

[16] Jonathan Strickland, How Cloud Computing Works. 